\title{
Central nervous system metastasis of an intradural malignant peripheral nerve sheath tumor in a dog
}

\author{
Federica Poli ${ }^{1, *}$, Maurizio Calistri ${ }^{1}$, Maria Teresa Mandara ${ }^{2}$ and Massimo Baroni ${ }^{1}$ \\ ${ }^{1}$ Valdinievole Veterinary Clinic, Via Costantino Nigra, Monsummano Terme (PT), Italy \\ ${ }^{2}$ University of Perugia, Department of Veterinary Medicine, Via San Costanzo, Perugia, Italy
}

\begin{abstract}
An 8-yr-old French Bulldog was presented with a non-ambulatory tetraparesis. Magnetic resonance showed an intradurally located mass at the level of the right second cervical nerve root. The mass was surgically removed and the dog was ambulatory within $4 \mathrm{~d}$. A 10-mo post-surgical imaging follow-up revealed a recurrence of the primary mass and another intradural/intramedullary mass at the level of the first thoracic vertebral body. Overall histological features were suggestive of malignant peripheral nerve sheath tumor (MPNST) for both masses. Immunohistochemistry was found weak but diffusely positive for S-100 and neurono-specific enolase for both masses. A diagnosis of primary MPNST for the cervical mass and of metastasis for the thoracic mass was made, possibly disseminated via the subarachnoidal space. To our knowledge, the central nervous system metastasis of MPNSTs has not previously been reported in dogs. The clinician should be aware that these tumors, albeit rarely, can metastasize to the central nervous system.
\end{abstract}

Keywords: Central nervous system metastasis, Dog, Malignant peripheral nerve sheath tumor.

\section{Introduction}

Peripheral nerve sheath tumors (PNSTs) are a group of neoplasms originating from Schwann cells, fibroblasts, or perineural cells (Summers et al., 1995). These tumors are classified into benign PNST (BPNST; e.g., schwannomas, neurofibromas) or malignant PNST (MPNST), mainly depending on the histological characteristics and aggressive behavior (Koestner and Higgins, 2017). MPNSTs are reported in various species and in various anatomic sites (Ramirez et al., 2007; Schulman et al., 2009; Steinmetz et al., 2010; Tavalsoy et al., 2013). In dogs, spinal nerves in the caudal cervical and cranial thoracic region and cranial nerves are most commonly affected. MPNSTs are described to be locally recurrent, and the overall long-term prognosis is considered to be poor (Brehm et al., 1995; Dolera et al., 2016; Hansen et al., 2016; Swift et al., 2017; Van Stee et al., 2017). Rarely, metastases of MPNSTs are reported in dogs (Stoica et al., 2001; Garcìa et al., 2004) or in other species, such as cows (Sartin et al., 1996), goats (Ramirez et al., 2007), and cats (Buza et al., 2012). In the two canine reports, the sites of metastases were lungs, spleen, liver, and kidneys. A recent study on canine soft-tissue sarcomas reported an $11.1 \%$ (27/242 cases) frequency of metastasis of PNST (Bray et al., 2014); one site of metastasis included the central nervous system, but it is not clarified whether it was due to an MPNST or to another type of soft-tissue sarcoma. In humans, MPNSTs have an incidence of $0.001 \%$ (Baehring et al., 2003) in the general population and develop more commonly in patients with neurofibromatosis type 1 (NF-1). In non-NF-1 patients, distant metastases are described. Sites of metastases included lungs, bone, pleura, retroperitoneum, diaphragm, lymph nodes, liver, chest wall, pericardium, thyroid gland, adrenal gland, leptomeninges, and brain. Spinal cord and brain metastases are reported to be very rare (Hruban et al., 1990; Baehring et al., 2003; Baek et al., 2005; Xu et al., 2012; Park et al., 2013; Ren et al., 2013; Thomas et al., 2014). In cattle, a form of multifocal benign neurofibroma is described involving mainly the brachial plexus, intercostal nerve, and cardiac nerve. A form of cutaneous multifocal neurofibroma resembling the human Von Recklinghausen disease was also described in cattle (Sartin et al., 1994). To the best of our knowledge, central nervous system metastases of MPNST have not yet been described in dogs or domestic animals; thus, we report the clinical, radiological, and histopathological findings of a rare occurrence of a central nervous system metastasis from an intradural MPNST in a dog.

\section{Case Details}

An 8-yr-old male French bulldog with a 1-month history of progressive tetraparesis was admitted to the clinic. $\mathrm{He}$ was administered steroids (prednisolone $0.5 \mathrm{mg} /$ $\mathrm{kg}$ ) for $20 \mathrm{~d}$ without improvement. In the neurological examination, the dog was alert and showed a severe non-ambulatory tetraparesis. Cranial nerves and spinal reflexes were judged within normal limit despite the aggressive behavior of the patient: the neurolocalization 
was consistent with a C1-C5 myelopathy. Possible differential diagnoses included a degenerative disease (disk herniation), an inflammatory-infectious disease (meningomyelitis and discospondylitis), neoplasia (primary or secondary) and, less likely, a congenital anomaly. Blood analysis showed no abnormality, nor did thoracic and cervical radiographs. The magnetic resonance imaging (MRI) scan (0.25T magnet, Vet MR Grande, Esaote Genoa) included T2W sagittal and transverse images, T1-weighted (T1W) sagittal and transverse images, and $\mathrm{T} 1 \mathrm{~W}$ post-intravenous contrast administration (gadopentate dimeglumine, $0.2 \mathrm{ml} / \mathrm{kg}$, Magnevist, Bayer). GE short TI inversion recovery images in the dorsal and transverse plain were also acquired. A large dumbbell-shaped intraduralextramedullary (golf tee-sign) paraspinal occupying space mass was seen at the level of the $\mathrm{C} 2$ vertebral body, severely compressing the spinal cord dorsally and laterally on the right side. The intraspinal component of the mass measured approximately 13 $\mathrm{mm}$ craniocaudally, $8 \mathrm{~mm}$ transversely, and $10 \mathrm{~mm}$ dorsoventrally and had an isointense signal in both $\mathrm{T} 1 \mathrm{~W}$ and T2-weighted (T2W) images, with strong and nearly homogeneous contrast enhancement (Fig. 1). The mass extended throughout the right C2 vertebral foramen, which appeared subjectively enlarged, infiltrating the C2 spinal nerve (dorsal branch or greater occipital nerve) through the paraspinal muscles. Atrophy of the obliquus capitis caudalis, semispinalis capitis, splenius capitis, and longus capitis muscles on the right side was present. Other lesions were not detected in the thoracolumbar spinal cord.

A brain scan was also obtained and did not show any abnormalities. The MRI findings were strongly suggestive of a PNST of the C2 nerve. The patient underwent surgery with a $\mathrm{C} 1-\mathrm{C} 2$ hemilaminectomy with marginal resection of the mass. The owner declined adjuvant radiotherapy.

Histopathology analysis showed a proliferation of spindle cells arranged in solid areas and occasional swirls scattered in myxoid to collagen matrix. The neoplastic cells had oval to elongated nuclei with finely stippled chromatin tending to become vesicular, as well as an unapparent nucleolus and eosinophilic cytoplasm. Nuclear atypia was marked, and mitotic figures were numerous [11 mitoses $\times 10$ high power field (HPF)]. Anisokaryosis and anisocytosis were marked. Gangliar and radicular elements were diffusely infiltrated by the neoplastic spindle cells. Immunohistochemistry analysis showed a positive stain for S-100 and neuronospecific enolase (NSE). Albeit with weak labeling, the immunoreaction appeared to involve more than $90 \%$ of neoplastic cells. Based on the histological findings, a diagnosis of MPNST was made.

The dog was ambulatory in a few days after the surgery and improved in the following weeks. The dog was re-admitted to the clinic after 7 mo because of a mild worsening of the neurological conditions and underwent an MRI scan. The follow-up MRI showed a partial regrowth of the intraspinal part of the mass. The patient worsened again in the following $3 \mathrm{mo}$, showing an ambulatory tetraparesis; the paresis was more marked in the hindlimbs, and the dog was mildly hypometric in the forelimbs. A third MRI scan was performed, which showed a nearly total regrowth of the intraspinal mass with a focal intramedullary $\mathrm{T} 2$ hyperintensity. An intramedullary/intradural-extramedullary lesion was also evident at the level of the T1 vertebral body, occupying a great part of the medullary diameter, extending cranially and caudally for a one-vertebral body length. The lesion had an isointense signal on both $\mathrm{T} 1$ and $\mathrm{T} 2 \mathrm{~W}$ images, with a $\mathrm{T} 2$ hyperintense signal at the cranial and caudal limits and showed a strong and homogeneous contrast enhancement at the T1 vertebral body level, with a contrast uptake of the ventral meninges to the $\mathrm{C} 7$ vertebral body (Fig. 2). The

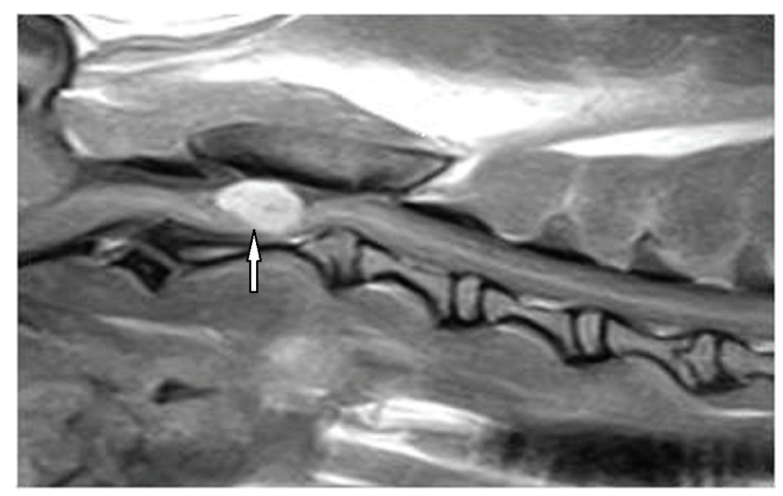

Fig. 1. A $T 1 \mathrm{~W}$ post-contrast sagittal image of the primary tumor, an intradural/extramedullary mass with homogeneous and strong contrast uptake (arrow).

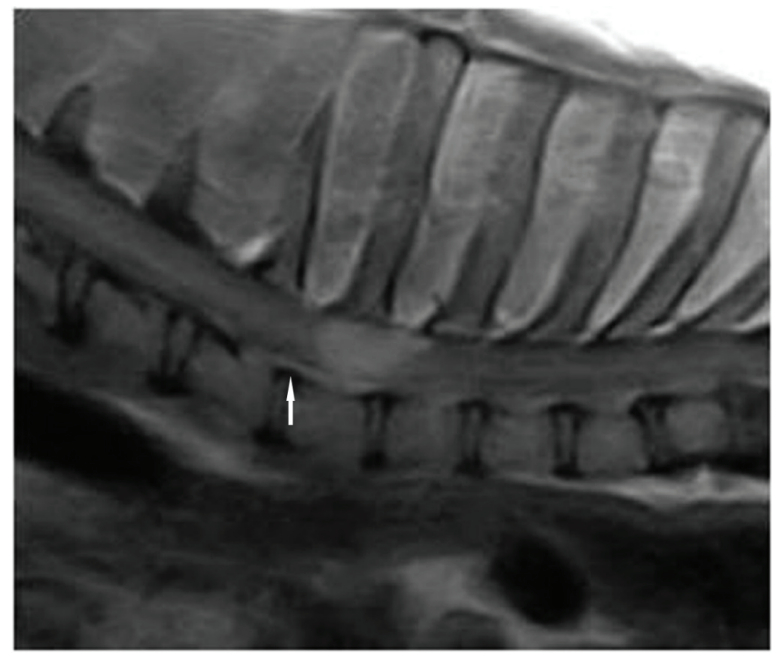

Fig. 2. A T1W post-contrast sagittal image of the cranial thoracic metastases of the C2 MPNST at the level of the first thoracic vertebral body, a well-contrast-enhanced intramedullary mass with meningeal involvement, as a "meningeal tail" extending cranially toward the C7 vertebral body (arrow). 
imaging findings were consistent with a recurrence of the C2 MPNST with intramedullary and meningeal metastasis at the cranial thoracic level. Thus, the owner elected euthanasia.

A post-mortem examination was performed and histopathology of the cervical and thoracic spinal cord confirmed the presence of PNSTs in both sites. However, compared to the previously resected tumor, a high mitotic rate (15/10 HPF) and neoplastic emboli in the meningeal blood vessels (Fig. 3) in both the recurrence site and the metastases were also seen. This neoplastic tissue again showed diffuse weak S-100 protein and NSE immunoreaction. The thoracic mass had a subdural-extramedullary localization severely compressing the spinal cord and producing a nearly total replacement of the gray matter. The neoplastic growth also affected the subdural emergences of the spinal nerves. A severe infiltration of the leptomeninges and medullary parenchyma with a perivascular pattern was observed. The histological findings confirmed an MPNST recurrence with spinal metastases (Fig. 4).

\section{Discussion}

MPNST central nervous system metastases and their features have not been reported in dogs. The radiological description of human MPNST spinal metastases from sporadic case reports is mainly intradural contrastenhancing lesions (Thomas et al., 2014) or multiple edematous infiltrations of the spinal cord in $\mathrm{T} 2 \mathrm{~W}$ images and multiple enhancing point lesions in $\mathrm{T} 1 \mathrm{~W}$ images (Park et al., 2013). Another report of human MPNST central nervous system metastasis showed the appearance of extensive leptomeningeal enhancement (Baek et al., 2005). In our case in a French bulldog, the MRI showed histologically confirmed metastases as a focal contrast-enhancing mass with an intradural/ intramedullary location, with meningeal involvement. In humans, three types of central nervous system

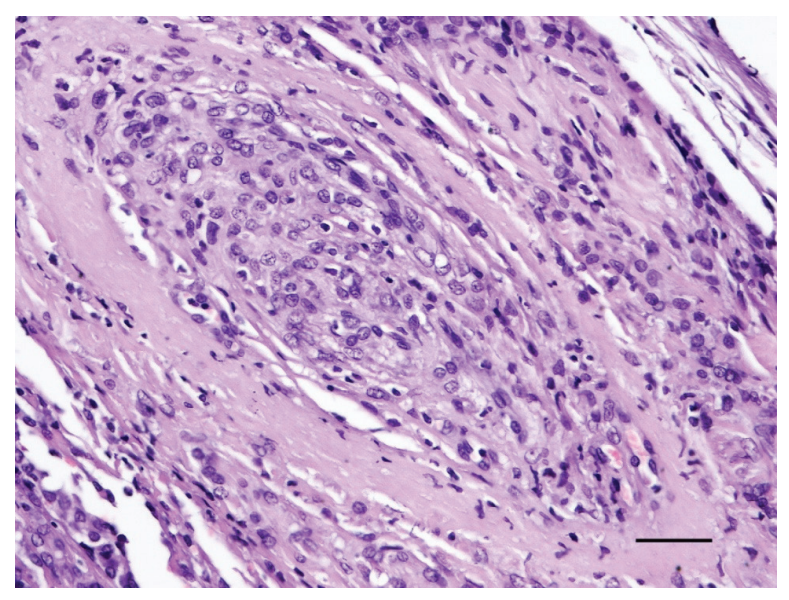

Fig. 3. The C2 spinal cord segment. A venous subarachnoid vessel is entirely occupied by neoplastic cells in association with subdural neoplastic infiltration $(\times 40 ; \quad \mathrm{H} \& \mathrm{E})$ (Bar: $30 \mu \mathrm{m}$ ). metastatic pathways of MPNST are described: direct invasion, cerebrospinal fluid dissemination, and hematogeneous metastases (Park et al., 2007). The histological examination of the cervical and thoracic masses in our case suggests a possible way of metastatic dissemination through the cerebrospinal fluid ("drop metastasis"), given the wide presence of cells and neoplastic tissue in the subarachnoid space; for this reason, an independent growth of neoplastic cells in each site (multifocal occurrence of the MPNST) has been considered unlikely.

The median survival times in dogs with root and peripheral nerve involvement is 5 and $12 \mathrm{mo}$, respectively, and the tumor relapse-free interval is 1 and 7.5 mo, respectively (Brehm et al., 1995; Lorentz et al., 2011); however, complete surgical resection can result in prolonged survival (Van Stee et al., 2017). A regrowth of the tumor and metastasis 7 and 10 mo after the surgery, respectively, in our patient could be either due to the microscopic residual neoplastic cells or the invasiveness of the tumor itself. In humans, the high recurrence and low survival rates can be explained by incomplete tumor removal; indeed, complete resection of intradural MPNST with tumor-free margins is very difficult due to the possibility of residual tumor cells remaining on surrounding dura mater and large blood vessels (Zhu et al., 2012). In a large human study on MPNST, Baehring et al. (2003) concluded that the involvement of proximal peripheral neural structures did not allow a total resection and lead to centripetal progression and infiltration of the spinal cord and brain stem. In humans, as in dogs, surgical excision with at least histologically clean margins is the goal of the treatment of MPNST; chemotherapy usefulness in humans is yet to be proven and is reserved for metastatic disease (Baehring et al., 2003; Park et al., 2013). A recent study suggests stereotactic radiotherapy as a promising alternative to surgical treatment in dogs,

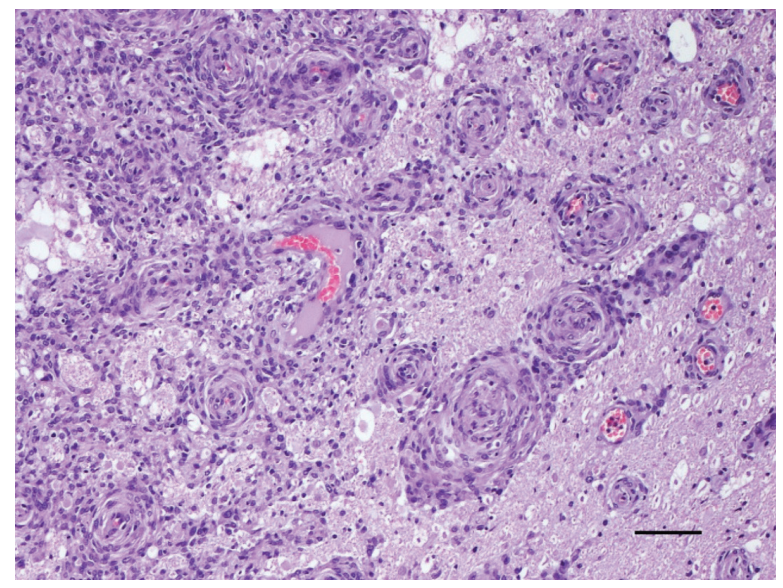

Fig. 4. The T2 spinal cord segment. The neoplastic cells infiltrate the nervous tissue from the leptomeninges through perivascular spaces, producing a perivascular whirl pattern $(\times 10$; H\&E) (Bar: $120 \mu \mathrm{m})$. 
at least for proximal and nerve root location (Dolera et al., 2016). Unfortunately, a complete post mortem examination was not done on our patient; thus, we do not know whether systemic hematogenous metastases were present, although possible given the presence of neoplastic emboli in the meningeal blood vessels.

This case report describes for the first time clinical, imaging, and histopathological findings of a cranial cervical region MPNST developing metastases to the central nervous system in a dog.

\section{Conflict of interest}

The authors declare that there is no conflict of interest.

\section{References}

Baehring, J.M., Betensky, R.A. and Batchelor, T.T. 2003. Malignant peripheral nerve sheath tumour: the clinical spectrum and outcome treatment. Neurology. 61, 696-698.

Baek, W.S., Pytel, P., Undevia, S.D. and Rubeiz, H. 2005. Spinal cord metastasis of a non-neurofibromatosis type-1 malignant peripheral nerve sheath tumour: an unusual manifestation of a rare tumour. J. Neuro. Oncol. 74, 183-185.

Bray, J.P., Polton, G.A., McSporran, K.D., Bridges, J. and Whitbread, T.M. 2014. Canine soft tissue sarcoma managed in first opinion practice: outcome in 350 cases. Vet. Surg. 43, 774-782.

Brehm, D.M., Vite, C.H., Steinberg, H.S., Haviland, J. and Van Winkle, T. 1995. A retrospective evaluation of 51 cases of peripheral nerve sheath tumours in the dog. J. Am. Anim. Hosp. Assoc. 31, 349-359.

Buza, E.L., Menzies, R.A., Goldschmidt, M.H. and Durham, A.C. 2012. Malignant peripheral nerve sheath tumour in a cat with nodal and pulmonary metastases. J. Vet. Diagn. Invest. 24, 781-784.

Dolera, M., Malfassi, L., Bianchi, C., Carrara, N., Finesso, S., Marcarini, S., Mazza, G., Pavesi, S., Sala, M. and Urso, G. 2016. Frameless stereotactic volumetric modulated arc radiotherapy of brachial plexus tumours in dogs: 10 cases. Br. J. Radiol. 90, 1069.

Garcìa, P., Sanchez, B., Sanchez, M.A., Gonzalez, M., Rollan, E. and Flores, J.M. 2004. Epithelioid malignant peripheral nerve sheath tumour in a dog. J. Comp. Pathol. 131, 87-91.

Hansen, K.S., Zwingerberger, A.L., Theòn, A.P., Pfeiffer, I. and Kent, M.S. 2016. Treatment of MRI-diagnosed trigeminal nerve sheath tumors by stereotactic radiotherapy in dogs. J. Vet. Intern. Med. 30(4), 1112-1120.

Hruban, R.H., Shiu, M.H., Senie, R.T. and Woodruff, J.M. 1990. Malignant peripheral nerve sheath tumours of the buttock and lower extremity. A study of 43 cases. Cancer. 66, 1253-1265.

Koestner, A. and Higgins, R.J. 2017. Tumors of the nervous system. In Tumors of Domestic Animals. Ed., Meuten, D.J., 5th ed. Ames, IA:Wiley Blackwell, pp: 842-891.
Lorentz, M.D., Coates, J.R. and Kent, M. 2011. Malignant peripheral nerve sheath tumors. In Handbook of Veterinary Neurology. 5th ed. Elsevier, pp: 103-104.

Park, M.K., Sung, J.K., Nam, K.H. and Kim, K.T. 2013. Malignant peripheral nerve sheath tumour of non-neurofibromatosis type 1 metastasized to the cerebrospinal axis. J. Korean Neurosurg. Soc. 53, 190-193.

Park, S.K., Yi, H.J., Paik, S.S., Kim, Y.J., Ko, Y. and Oh, S.J. 2007. Metastasizing malignant peripheral nerve sheath tumor initially presenting as intracerebral hemorrhage. Case report and review of the literature. Surg. Neurol. 68, 79-84.

Ramirez, G.A., Herraez, P., Rodriguez, F., Godhino, A., Andrada, M. and de los Menteros, A.E. 2007. Malignant peripheral nerve sheath tumour (malignant schwannoma) in the diaphragm in a goat. J. Comp. Pathol. 137, 137-141.

Ren, X., Wang, J., Hu, M., Jiang, H., Yang, J. and Jiang, Z. 2013. Clinical, radiological, and pathological features of 26 intracranial and intraspinal malignant peripheral nerve sheath tumours. J. Neurosurg. 119, 695-708.

Sartin, E.A., Doran, S.E., Riddell, M.G., Herrera, G.A., Tennyson, G.S., D'Andrea, G., Whitley, R.D. and Collins, F.S. 1994. Characterization of naturally occurring cutaneous neurofibromatosis in Holstein Cattle. A disorder resembling neurofibromatosis type 1 in humans. Am. J. Pathol. 145, 1168-1174.

Sartin, E.A., Herrera, G.A., Wilkins, L.P. and Wolfe, D.F. 1996. Metastatic multicentric neurofibrosarcoma of the lombosacral plexus in a cow. Vet. Pathol. 33, 362-365.

Schulman, F.Y., Johnson, T.O., Facemire, P.R. and Fanburg-Smith, J.C. 2009. Feline peripheral nerve sheath tumors: histologic, immunohistochemical, and clinicopathologic correlation (59 tumors in 53 cats). Vet. Pathol. 46, 1166-1180.

Steinmetz, H.W., Rutten, M., Ruess-Melzer, K., Ohlerth, S., Lischer, C., Oevermann, A., BodeLesniewska, B. and Hatt, J.M. 2010. Clinical course of a malignant peripheral nerve sheath tumor in a Siberian tiger (Panthera tigris altaica). J. Vet. Diagn. Invest. 22, 970-975.

Stoica, G., Tasca, S.I. and Kim, H.T. 2001. Point mutation of neu oncogene in animal peripheral nerve sheath tumours. Vet. Pathol. 38, 679-688.

Summers, B.A., Cummings, J.F. and de Lahunta, A. 1995. Neoplasia and the peripheral nervous system. In Veterinary Neuropathology. St Louis, MO: Mosby, pp: 472-481.

Swift, K.E., McGrath, S., Nolan, M.W., Young, M., Reese, M., Rao, S., Randall, E., Leary, D. and LaRue, S. 2017. Clinical and imaging findings, treatments, and outcomes in 27 dogs with imaging diagnosed trigeminal nerve sheath tumors: a 
multi-center study. Vet. Radiol. Ultrasound. 58(6), 679-689.

Tavalsoy, A., Javanbakht, J., Khaki, F., Hosseini, E., Bahrami, A., Hassan, M.A. and Mirabad, M. 2013. Ulnar malignant peripheral nerve sheath tumour diagnosis in a mixed-breed dog as a model to study human: histologic, immunohistochemical, and clinicopathologic study. Diagn. Pathol. 8, 86.

Thomas, J.G., Lincoln, C., Goodman, J.C. and Gopinath, S.P. 2014. Malignant peripheral nerve sheath tumour of the cauda equina with craniospinal metastasis. J. Clin. Neurosci. 21, 2239-2242.

Van Stee, L., Boston, S., Teske, E. and Meij, B. 2017. Compartmental resection of peripheral nerve tumours with limb preservation in 16 dogs (19952011). Vet. J. 226, 40-45.

Xu, Q., Xing, B., Huang, X., Wang, R., Li, Y. and Yang, Z. 2012. Primary malignant nerve sheath tumor of the cauda equina with metastasis to the brain in a child: case report and literature review. Spine J. 12, e7-e13.

Zhu, B., Liu, X., Liu, Z., Yang, S., Liao, H.I., Jiang, L. and Wei, F. 2012. Malignant peripheral nerve sheath tumours of the spine: clinical manifestation, classification, treatment, and prognostic factors. Eur. Spine J. 21, 897-904. 